\title{
SILAC Quantification
}

National Cancer Institute

\section{Source}

National Cancer Institute. SILAC Quantification. NCI Thesaurus. Code C161817.

A proteomic quantitation method where a sample is produced using stable isotope

labeling with amino acids in cell culture (SILAC) before being subjected to protein

digestion and mass spectrometry-based quantification analysis. 